\title{
Microfabrication of conical microfunnels for drug delivery applications
}

\author{
L.W.Y. Liu', B.S. Virdee', J. Inal ${ }^{3}$, M.B. Steer ${ }^{4}$ \\ ${ }^{1}$ Green Education For Life, 18, Lane 8, Po Sheung Village, Sheung Shui, NT, Hong Kong \\ ${ }^{2}$ Centre for Communications Technology, London Metropolitan University, London N7 8DB, UK \\ ${ }^{3}$ Cellular and Molecular Immunology Research Centre, London Metropolitan University, London N7 8DB, UK \\ ${ }^{4}$ Department of Electrical and Computer Engineering, College of Engineering, 435 Monteith, Campus Box 7911, NC State \\ University, Releigh, NC, 27695-7911, USA \\ E-mail: planetx4u@yahoo.com
}

Published in Micro \& Nano Letters; Received on 4th December 2014; Revised on 30th April 2015; Accepted on 30th April 2015

\begin{abstract}
A cost-effective fabrication methodology for the mass production of conical microfunnels for drug delivery applications is presented. The fabrication methodology originates from a technique previously used for fabricating suspended or hollow microstructures. The process comprises six stages: in stage 1, a UV-opaque resin substrate is formed by mixing photocurable SU-8 with a UV-opaque impurity; in stage 2, an embossing stamp with an array of microrods is fabricated; in stage 3, the upper surface of the UV-opaque resin substrate (from the first stage) is mechanically deformed by stamping with the embossing stamp from stage 2 ; in stage 4 , the regions in which microfunnels are photolithographically defined are selectively exposed to UV light; in stage 5, the wafer from stage 4 is subjected to heat and then developed in 1-methoxy-2-propanol acetate; finally, a hole on the tip of each microfunnel is formed by dry etching with oxygen plasma. The sharpness of the funnel tips can be flexibly adjusted by changing the stroke speed of the stamping in stage 3 . The overall process can be carried out photolithographically with conventional photolithographic equipment. Using the proposed fabrication methodology, a matrix of polymeric microfunnels tapering from the base measuring $300 \mu \mathrm{m}$ to the tip measuring $50 \mu \mathrm{m}$ over a funnel height of $100 \mu \mathrm{m}$ has been fabricated. Each fabricated microfunnel features a wide base and a sharp tip so that it has sufficient mechanical strength and sharpness to pierce the human skin. The conical geometry used in this study had a volume in excess of $24.4 \mathrm{nl}$.
\end{abstract}

1. Introduction: Over the last few decades, there has been no shortage of interest in microfabricated drug delivery systems. Most of these microfabricated drug delivery systems are based on an array of hollow microneedles fabricated in micron resolution. However, the formation of hollow objects at such a high resolution is one of the well-known challenges in the field of microfabrication. As yet, there has not been any cost-effective fabrication approach dedicated to the mass production of hollow microstructures at micron resolution without microinjection moulding. This Letter presents an enabling technology for the realisation of conical microfunnels for drug delivery applications. The proposed fabrication methodology originates from a technique previously used for fabricating suspended or hollow microstructures [1-4]. Unlike other three-dimensional (3D) microfabrication technologies, the proposed fabrication methodology is based on photolithography only and it can be carried out with conventional photolithographic equipment.

2. Concept behind the proposed fabrication methodology: Figs. $1 a-d$ illustrate the concept behind the proposed fabrication methodology.

Like any other negative photosensitive resin, a pure SU-8 should be UV transparent. However, when a pure SU-8 is mixed with a UV-opaque pigment or metallic nanoparticles, as shown in Fig. 1 $a$, it loses its UV transparency. When this bulk of UV-opaque SU-8 undergoes a prolonged dehydration bake, it becomes highly viscous and its surface becomes non-adhesive.

The non-adhesive surface of the UV-opaque SU-8 mass is then mechanically deformed by an impact produced by a sharpened object, as illustrated in Fig. $1 b$, while the wafer is being cooled down. As a result of this impact, a valley is formed on the upper surface of the UV-opaque SU-8 mass, where the steepness of the valley depends on the stroke speed of the impact and the temperature of the wafer. Since the UV-opaque SU-8 sample is non-elastic, it does not bounce back.
In the presence of the UV-opaque impurity, the SU-8 mass has obviously lost its UV-transparency. However, losing its UV transparency does not necessarily mean the absence of any photopolymerisation. When exposed to UV-light, as illustrated in Fig. 1c, the UV light can still reach the upper surface and the penetration depth depends on the duration of UV exposure. The surface area on which the UV light has shone will be polymerised into a thin layer. The thickness of this layer will depend on how long the UV-opaque SU-8 sample has been exposed to UV light.

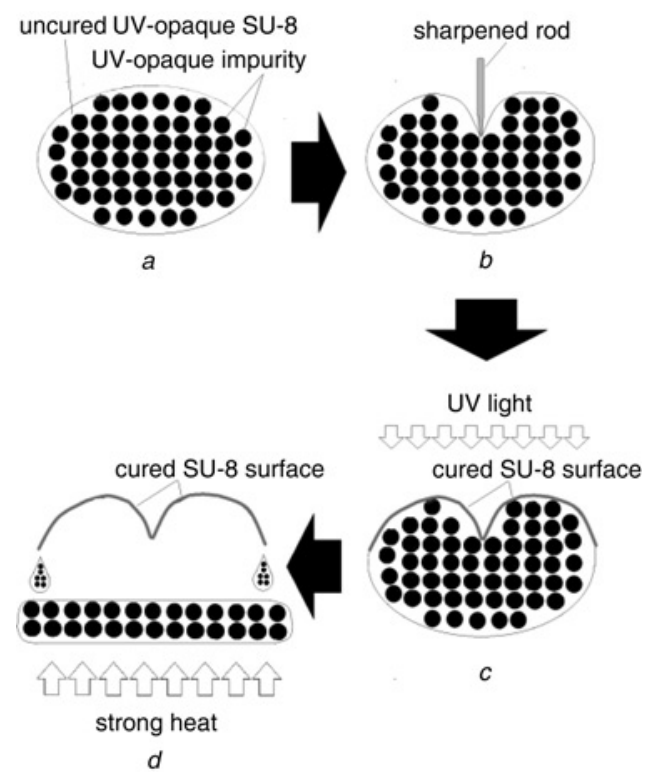

Figure 1 Cross-sectional views that illustrate how a highly dehydrated $U V$-opaque $S U-8$ behaves when exposed to UV light 
The UV-opaque SU-8 resin underneath the UV-exposed surface will remain uncured. When subjected to heat, as shown in Fig. 1d, the surface area having exposure to the UV light becomes further cross-linked and hardened. However, since the UV-opaque SU-8 underneath the UV-exposed surface remains uncured, it will melt into a liquid when subjected to heat. The UV-opaque SU-8 that remains underneath the polymerised surface can be stripped off by developing in 1-methoxy-2-propanol acetate.

3. Preparation of a UV-opaque $\mathbf{S U - 8}$ resin: To start with, we require a pre-polymerised SU-8 which is not only UV curable but also UV-opaque. To realise this UV-opaque SU-8, a pure SU-8 monomer resin is pre-mixed with a UV-opaque impurity until the final mixture becomes almost opaque in the UV spectrum. This UV-opaque impurity can be extracted from any organic chemical that is not only opaque to UV light but also highly soluble in SU-8. Our experiment suggests that the pigment in synthetic rubber Blu Tack ${ }^{\mathrm{TM}}$ is both soluble in SU-8 and UV-opaque.

After the mixing process, the UV-opaque SU-8 resin undergoes a prolonged dehydration bake. This baking process not only increases the viscosity of the UV-opaque SU-8 resin but also decreases its surface adhesion. This UV-opaque SU- 8 resin should be able to reshape on heating and retain its physical and chemical properties after cooling down.

4. Fabrication of an embossing stamp: An embossing stamp is a master mould which serves as a patterned template for casting of the desired 3D patterns on the surface of a UV-opaque photosensitive resin. As schematically illustrated in Fig. 2, the embossing stamp to be formed consists of an array of microrods fabricated in SU-8 or metal. A variety of methods can be used to form this embossing stamp. We fabricated an embossing stamp with high aspect ratio microrods using a high-quality standard UV-lithographic process.

5. Fabrication of microwells: Once the embossing stamp and the UV-opaque SU-8 resin are available, fabrication of hollow or suspended microstructures can proceed as shown in Figs. $3 a-d$.

In step 1, as shown in Fig. $3 a$, several height-defining blocks of equal height are fabricated onto the corners of the substrate. These height-defining blocks are used to ensure that the SU-8 resin to be deposited on the wafer becomes even and accurate in thickness.

In step 2, as shown in Fig. 3b, the UV-opaque SU-8 resin is deposited onto the surface of the substrate until the thickness of the UV-opaque SU-8 resin slightly exceeds the height of the heightdefining blocks. The wafer is then heated slightly above the glass-transition temperature for a prolonged period until the UV-opaque SU-8 resin becomes partially molten and its upper surface becomes non-adhesive.

In step 3, as shown in Fig. 3c, the top surface of the UV-opaque $\mathrm{SU}-8$ resin is pressurised and ironed flat with a glass plate. Then, the heat source is removed.

In step 4, as shown in Fig. $3 d$, the upper surface of the UV-opaque SU-8 is mechanically deformed by an impact produced by the embossing stamp moving downwards at high speed. At the same time, the wafer is being cooled down. As a result of this impact, microconical wells are three-dimensionally formed on the

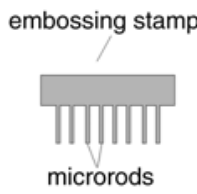

Figure 2 Cross-sectional view that illustrates the embossing stamp with microrods

Diameter of each microrod is $40 \mu \mathrm{m}$

Length of each microrod is $200 \mu \mathrm{m}$

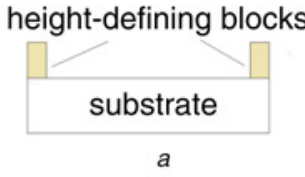

UV-opaque SU-8
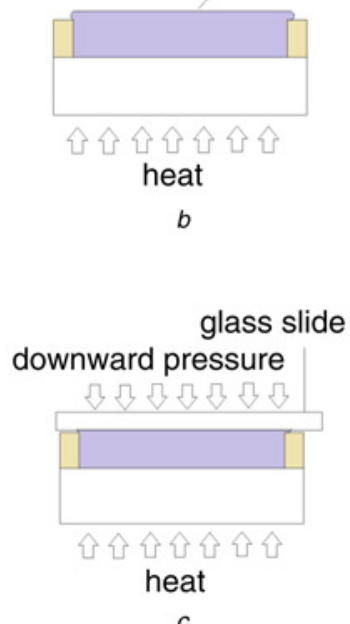

embossing stamp

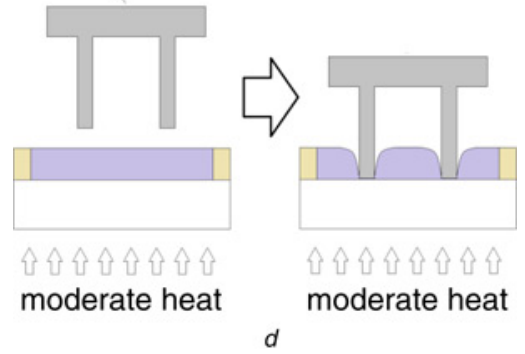

Figure 3 Process for the formation of conical microwells (cross-sectional view)

upper surface of the UV-opaque SU-8. The stroke speed of the stamping will determine the surface profile of the inner wall and the sharpness of the tip of each conical well. In general, the tip of each conical well will become sharper if the speed of the stamping is higher.

In step 5, as shown in Fig. $3 d$, the embossing stamp is removed from the wafer. The wafer is then cooled down until the conical microwells become highly solidified.

6. Increasing the shell thickness by increasing the UV exposure: As shown in Fig. 4, the wafer with the microwell patterns is photolithographically patterned and exposed to UV light using a photomask. Since the SU-8 resin has been pre-mixed with a

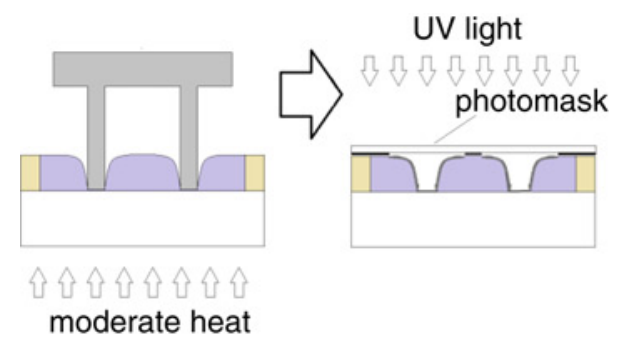

Figure 4 Formation of conical funnels by UV-exposure (cross-sectional view) 


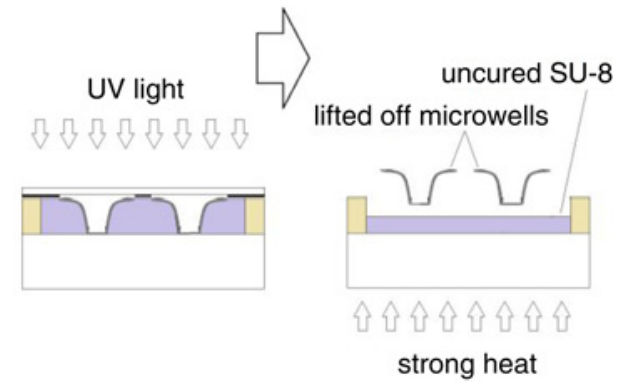

Figure 5 Lifting off the conical funnels by melting away the uncured $S U-8$ underneath the conical funnels

UV-opaque impurity, it is basically opaque to UV lights. In the presence of the UV-opaque impurity, the inner surface of each microwell will be polymerised into a hard layer while the SU-8 resin underneath the UV-exposed regions will remain uncured. The thickness of this polymeric layer can be easily changed by changing the duration of UV exposure. In general, the longer the UV exposure time, the thicker this polymeric layer will become. This polymeric layer can be as thin as a membrane or as thick as the application demands.

7. Melting off the uncured UV-opaque SU-8 resin: The SU-8 resin underneath the UV-exposed surface will remain uncured and become removable after melting. As shown in Fig. 5, the wafer is subjected to strong heat. The uncured SU-8 resin underneath the UV-exposed surface will be melted into a liquid, while the UV-exposed surface will be hardened. Traces of the remaining uncured photosensitive resin can be stripped off by 1-methoxy-2-propanol acetate.

8. Opening a hole on the tip of each conical microwell by dry etching: To turn each conical microwell into a microfunnel, we need to open a hole in the tip of each conical well. This step can be achieved by dry etching in oxygen plasma for $200 \mathrm{~s}$ using a Trion RIE/PECVD tool. The process parameters chosen for this work was $90 \% \mathrm{O}_{2}, 10 \% \mathrm{CF}_{4}$, an $\mathrm{RF}$ power of $100 \mathrm{~W}$ and a chamber pressure of 1.6 Torr. The oxygen plasma also simultaneously sharpens the tip of each microfunnel.

9. Results and discussion: Fig. 6 illustrates one of the microfunnels that we fabricated using the above-mentioned method. The microfunnels were designed to have a wide base and a sharp tip, which allowed a larger amount of drug encapsulated per microfunnel. The conical geometry used in this work had a volume in excess of $24.4 \mathrm{nl}$. By using low aspect ratio geometry, the microfunnels were designed to have sufficient mechanical strength for inserting into the human skin. The smallest tip diameter that has been achieved in this work is within $50 \mu \mathrm{m}$, which is sharp enough to pierce the human skin. We anticipate that, by shrinking the sizes, the proposed fabrication methodology can be further extended to fabricate other microneedle-based systems.

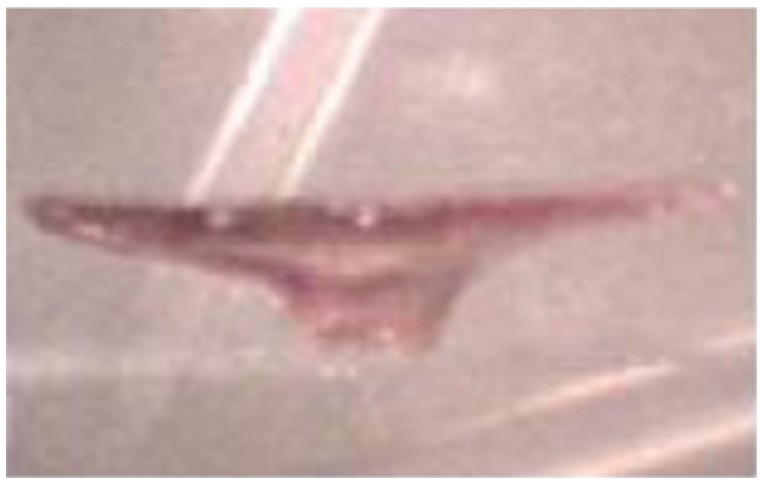

Figure 6 Photograph of one of the conical microfunnels fabricated using the proposed technique

10. Conclusion: This Letter has presented a method that enables an array of microfunnels to be photolithographically fabricated with conventional photolithographic equipment. The process comprises six stages, that is, in stage 1, a UV-opaque resin substrate is formed by mixing photocurable SU-8 with a UV-opaque impurity; in stage 2, an embossing stamp with an array of microrods is fabricated; in stage 3 , the upper surface of the UV-opaque resin substrate (from the first stage) is deformed by stamping with the embossing stamp from stage 2; in stage 4, the regions in which microfunnels are photolithographically defined are selectively exposed to UV light; in stage 5, the wafer from stage 4 is subjected to heat and then developed in 1-methoxy-2-propanol acetate, and finally, a hole on the tip of each microfunnel is formed by dry etching with oxygen plasma. The sharpness of the funnel tips can be flexibly adjusted by changing the stroke speed of the stamping in stage 3 . Using the proposed fabrication methodology, we have fabricated a matrix of conical microfunnels tapering from the base measuring $300 \mu \mathrm{m}$ to the tip measuring $50 \mu \mathrm{m}$ over a funnel height of $100 \mu \mathrm{m}$. The conical geometry used in this work had a volume in excess of $24.4 \mathrm{nl}$. By using low aspect ratio geometry, the microfunnels are designed to have sufficient mechanical strength for inserting into the human skin. The smallest tip diameter that has been achieved in this work is within $50 \mu \mathrm{m}$, which is sharp enough to pierce the human skin.

\section{References}

[1] Liu W.Y.: 'Photolithographical micro fabrication of hollow three dimensional structures with profiled side walls'. UK Patent Application GB2416599, February 2006

[2] Liu W.Y.: 'Micro machining process for forming three dimensional micro structures having sloping and profiled side walls'. UK Patent Application GB 2404454A, February 2005

[3] Virdee B.S., Liu W.Y.: 'On-chip cooling of RFICs and MICs'. 34th European Microwave Conf., Amsterdam, The Netherlands, October 2004

[4] Liu L.W.Y.: 'Fabrication of three dimensional hollow or suspended microstructures'. 2014 Int. Symp. on Micro-NanoMechatronics and Human Science (MHS2014), November 2014 